\title{
Vortex Shedding from a Circular Cylinder with Spiral Fin*
}

\author{
Hiromitsu HAMAKAWA ${ }^{* *}$, Keisuke NAKASHIMA ${ }^{* * *}$, Tomohiro KUDO ${ }^{* * * *}$, \\ Eiichi NISHIDA $^{* * * * *}$ and Tohru FUKANO ${ }^{* * * * * *}$ \\ **Department of Mechanical Engineering, Oita University, \\ 700 Dannoharu, Oita 870-1192, Japan \\ E-mail: hamakawa@cc.oita-u.ac.jp \\ ${ }^{* * *}$ Graduate Student, Oita University, \\ 700 Dannoharu, Oita 870-1192, Japan \\ **** TOTO Ltd, \\ 2-1-1 Nakashima, Kokurakita, 802-8601 Kitakyushu, Japan

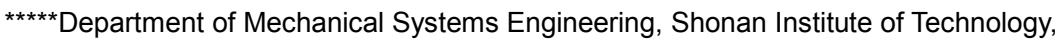 \\ 1-1-25 Tujidou Nisikaigan, Fujisawa 251-8511, Japan \\ ******Department of Environmental Symbiosis Engineering, Kurume Institute of Technology, \\ 2228 Kamitsu-machi, Kurume 830-0052, Japan
}

\begin{abstract}
The effect of simple spiral fin around a bare tube on Karman vortex shedding was experimentally investigated. We measured the distributions of the mean velocity, the intensity of velocity fluctuation, the spectrum of velocity fluctuation and the coherence distribution of Karman vortex in the spanwise direction. And the flow around a fin tube was visualized by using smoke wire technique. As a result it was made clear that the periodic velocity fluctuation caused by Karman vortex was remarkably observed in the wake of the fin tube although the fin was mounted around a bare tube with a large pitch along the tube axis. The vortices in the near-wake of the fin tube make cells structure with the scale of the pitch length of the fins. The rotation axis of each vortex was inclined against the tube axis. The large scale vortex was formed by the coalescence of the vortex cells. Therefore, the spanwise scale of the vortex in the wake of the fin tube was larger than one pitch of the fins.
\end{abstract}

Key words: Vortex, Spiral Fin, Spanwise Vortex Scale, Vortex Cells, Flow Visualization

\section{Introduction}

Aeolian tones are the sounds produced by vortex shedding from a circular cylinder. These tones can induce vibration in structures exposed to sound pressure. If the frequency of vortex shedding from tubes in a heat exchanger coincides with the natural frequency of the heat exchanger cavity, the sound of acoustic resonance can become so intense that it damages the heat exchanger. The far field sound power of Aeolian tone depends on the spanwise correlation length of vortex shedding from a circular cylinder ${ }^{(1)}$. Therefore, if the correlation length of vortex shedding is less than that of a circular cylinder, the excitation energy of acoustic resonance is reduced from the case of a circular cylinder.

It is generally considered that vortex induced vibration can be suppressed by mounting helical strakes around a bare tube ${ }^{(1)(2)}$. Zdravkovich ${ }^{(2)}$ have shown that helical strakes or several fins as surface protrusions around a bare tube have the effects of vibration reduction. 
Recently, fin tubes are used more frequently in heat exchangers to enhance heat transfer. The fins are mounted spirally around a bare tube surface because of easy production. However, loud resonant noise is occasionally generated in the duct when gas flows laterally to the axis of fin tube banks. Some studies have been reported on the characteristics of acoustic resonance and vortex shedding generated from fin tube banks. Nemoto et al. ${ }^{(3) \sim(5)}$ suggested that the acoustic resonance was generated even in fin tube banks. Chen ${ }^{(6)}$, Kouba ${ }^{(7)}$, Hamakawa et al. ${ }^{(8)}$, Kawaguchi et al. ${ }^{(9)}$ and Ziada et al. ${ }^{(10)}$ have shown that the vortex shedding was remarkably observed in fin tube banks or in the wake of a single fin tube. Mair et al. ${ }^{(11)}$, Suzuki et al. ${ }^{(12)}$, Hamakawa et al. ${ }^{(13)}$ and Ziada et al. ${ }^{(10)}$ have reported that the vortex shedding frequency was correlated with the tube effective diameter.

On the other hand, it is known that Karman vortex has three-dimensional correlation structure in the span direction even in the two-dimensional flow field. Therefore, it is considered that the vortex is cut easily by the fins and the vortex structure in the wake of a fin tube is different from a bare tube. Hamakawa et al. ${ }^{(13)}$ found that the spanwise scale of the vortex to be considerably larger than the pitch of the fins. Ziada et al. ${ }^{(10)}$ have shown that the correlation length in the wake of the serrated fin tube was found to change with angular rotation of the tube around its axis. This phenomenon seems to be related to an irregular wavy pattern of the serrated fin distribution along the tube axis. However, there are a few studies on the characteristics of vortex shedding from a fin tube, and the mechanism of the increase of correlation length of vortex is not clear. The spanwise correlation length of vortex shedding is the basic parameter that is needed to estimate the characteristics of unsteady fluid forces and sound generation by vortex shedding from a fin tube.

The purpose of the present paper is to clarify the effect of the simple spiral fin around a bare tube on the flow and vortex shedding. This fin was made from a thin plate and mounted spirally around a bare tube surface. The mechanism of the increase of the correlation length of Karman vortex shedding will be discussed.

\section{Experimental Apparatus and Procedures}

The schematic view of the experimental apparatus used in the present experiment is shown in Figure 1. The wind tunnel is an open-circuit with the blower located upstream of the test section. The cross section of nozzle exit is a square of $0.5 \mathrm{~m}$ wide and $0.5 \mathrm{~m}$ high. A fin tube is installed in the test section so that the front of the fin tube is $250 \mathrm{~mm}$ downstream of the nozzle exit.

The freestream velocity $U_{\infty}$ ranges from $5 \mathrm{~m} / \mathrm{s}$ to $29 \mathrm{~m} / \mathrm{s}$ at the test section inlet. Reynolds numbers, based on diameter $D$ and the freestream velocity $U_{\infty}$, ranges from $1.1 \times 10^{4}$ to 6.3 $\times 10^{4}$. The incoming flow from the nozzle is spatially uniform at the test section inlet and the freestream turbulence level is less than 0.52 percent at the freestream velocity range from 5 to $29 \mathrm{~m} / \mathrm{s}$. And no peak of spectrum of velocity fluctuation at the test section without the fin tube is formed at this velocity range. The length of the end plates of a test piece is

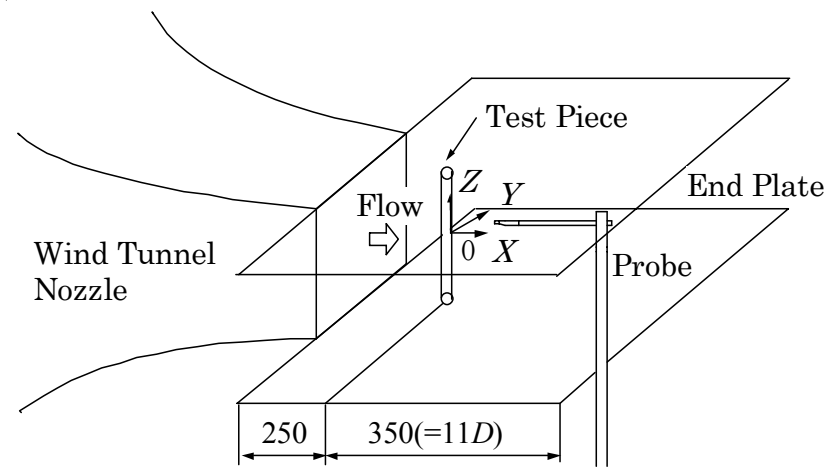

Fig. 1 Experimental apparatus and co-ordinate axes 
$350 \mathrm{~mm}(=11 \mathrm{D})$ from the trailing edge of the fin tube to the downstream.

Figure 2 shows a photograph of the fin tube. This fin is made from a plate with $1.2 \mathrm{~mm}$ thickness. The fin is mounted spirally around a bare tube surface. Bare tube diameter $D$ is about $31.8 \mathrm{~mm}$, fin diameter $D_{f}$ is about $57.2 \mathrm{~mm}$ and fin pitch $p$ are 32.0, 25.4, 8.47, 5.08 $\mathrm{mm}$, i.e., the ratios of fin pitch per tube diameter $p / D$ are $1.00,0.80,0.27,0.16$, which are presented in Table 1. These fins are mounted with an angle of 26.6, 21.8, 7.6, 4.6 degree against the main flow respectively. The span of the tube is $500 \mathrm{~mm}$. The fins are distributed regularly along the tube axis, which have not the irregular wavy pattern like the serrated fin distribution along the tube axis ${ }^{(10)}$. These fin tubes have the same geometry as those actually used in boiler tubes.

Mean velocity and velocity fluctuation in the wake of the tube are measured by using two I-type hot-wire sensors, with tungsten wires of $5 \mu \mathrm{m}$ in diameter and $1.25 \mathrm{~mm}$ in sensor length. The hot-wire sensors are directed in parallel with the span ( $Z$-axis) direction to measure the velocity fluctuation due to vortex shedding from the tube. One of the hot-wire sensors is traversed and fixed at a pre-determined location in the wake of the fin tube. The characteristics of flow, such as velocity distribution of mean flow and turbulence intensity, and the spectrum of the velocity fluctuation are measured on the $X-Y$ plane at different positions in the wake of the fin tube. The coefficient of velocity fluctuation $u$ ' is defined as the ratio of the intensity $\Delta u$, i.e. the standard deviation of velocity fluctuation, to the main flow velocity $U_{\infty}$, i.e., $\Delta u / U_{\infty}$.

The other hot-wire probe is installed when the cross correlation between the velocity fluctuations at two different locations is measured. The outputs from these hot-wire anemometers are automatically sampled by a computer and a FFT analyzer and then the statistical parameters are calculated. The hot-wire signals are digitized with sampling frequency of $512 \mathrm{~Hz}$. Fourier power spectra of signals are based on the average of 80 runs, each run composed of 2048 samples. The frequency resolution is estimated to be $0.25 \mathrm{~Hz}$.

The flow around the fin tube is visualized by using smoke wire technique, involving a Nichrome wire of $0.2 \mathrm{~mm}$ in diameter. A digital video camera is used to photograph the

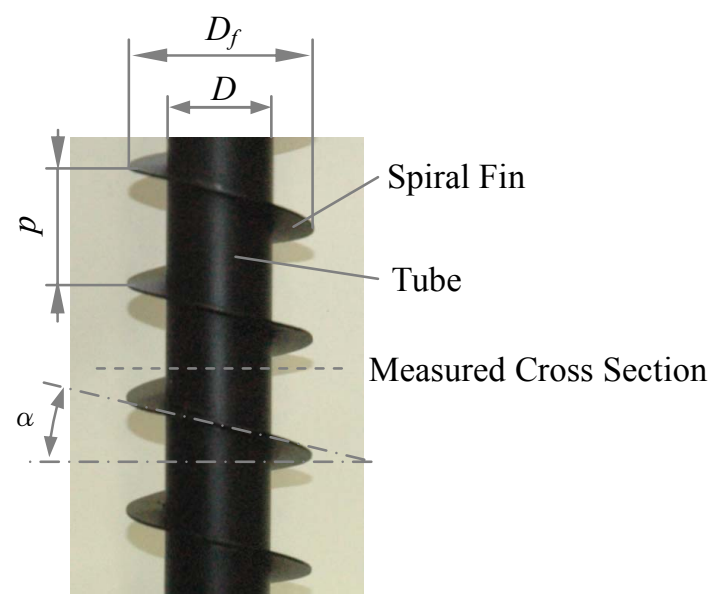

Fig. 2 Shape of a fin tube

Table 1 Specifications of a circular cylinder with fin

\begin{tabular}{|c|c|c|c|}
\hline$p / \mathrm{D}$ & $D_{f} \mathrm{~mm}$ & $D \mathrm{~mm}$ & $\alpha$ deg \\
\hline 1.00 & 57.0 & 32.0 & 26.6 \\
\hline 0.80 & 59.2 & 31.8 & 21.8 \\
\hline 0.27 & 57.2 & 31.8 & 7.6 \\
\hline 0.16 & 57.2 & 31.8 & 4.6 \\
\hline
\end{tabular}




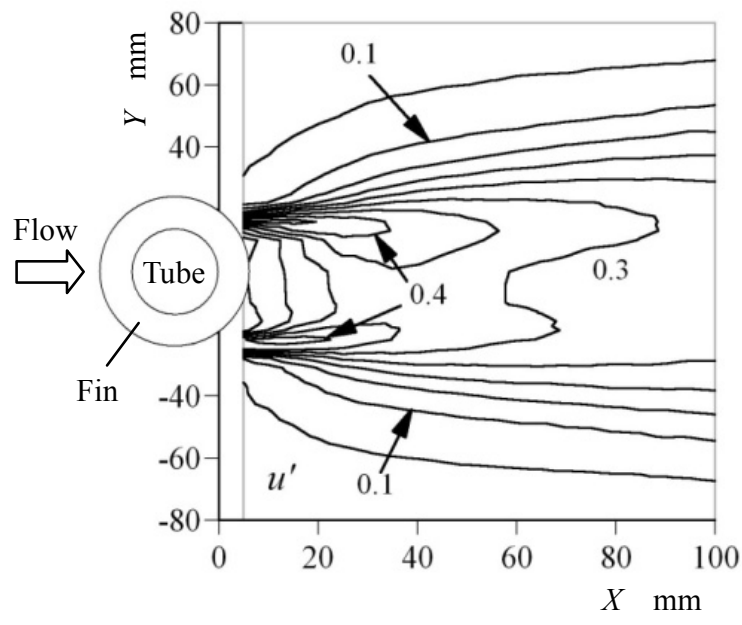

(a) Contour map of $u^{\prime}(p / D=1.00)$

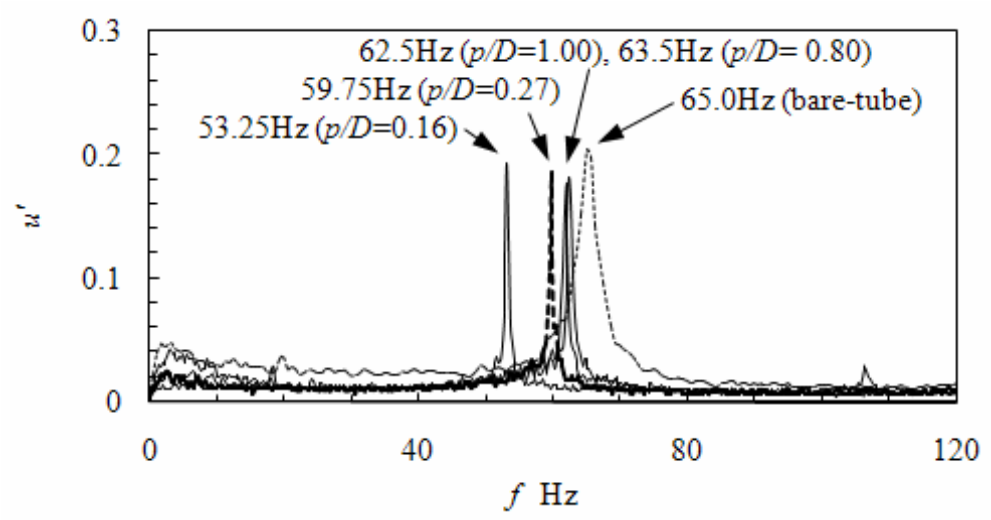

(b) Spectral distribution of $u^{\prime}{ }_{\text {max }}\left(U_{\infty}=10.0 \mathrm{~m} / \mathrm{s}\right)$

Fig. 3 Characteristics of velocity fluctuation in the wake of the fin tube

vortex shedding, which can process up to 30 frames per second. This camera is mounted at the outside of the test section. Visualizations are made at Reynolds number $2.1 \times 10^{3}$. These Reynolds numbers mentioned above are almost the same as the operating conditions of actual boiler plants.

\section{Results and Discussion}

\subsection{Vortex Shedding Characteristics from Spiral Fin Tube}

The fin tube is installed in the test section $250 \mathrm{~mm}$ downstream of the nozzle exit as shown in Figure 1. We have measured the intensity and the spectrum of velocity fluctuation on the $X-Y$ plane in the wake of the fin tube under the condition of freestream velocity of $10.0 \mathrm{~m} / \mathrm{s}$. The measured cross section is at the midpoint between the neighboring fins along the tube axis as shown dotted line in Fig. 2. The distribution of turbulence intensity in the wake of the fin tube of $p / D=1.00$ is shown in Fig. 3(a) as a contour map of $u^{\prime}$. The double circle in the figure shows the location of the fin tube on the $Y$-axis. There are two regions in which the velocity fluctuation is intense on the both sides just downstream of the fin tube in Fig. 3(a). Overall distribution of $u^{\prime}$ is symmetric with respect to the $X$-axis $(Y=0)$.

Figure 3(b) shows the spectrum of velocity fluctuation at its maximum point shown in Fig. 3(a). In Fig. 3(b) the spectrum in the wake of the fin tube is expressed as $p / D=1.00$. A single high peak is found at $62.5 \mathrm{~Hz}$ although the pitch of the fin is pretty large, which suggests the existence of the periodic phenomenon in the wake of the fin tube. The phase delay between the velocity fluctuations of two points of the intense velocity fluctuation on 
the both sides just downstream of the fin tube is 180 degree. This means that the velocity fluctuations are out of phase with each other.

Figure 4 shows the peak frequency of the spectrum of velocity fluctuation plotted against the freestream velocity. In Fig. 4 the peak frequency in the wake of the fin tube of $p / D=1.00$ is expressed as cross symbols, which is measured at maximum velocity fluctuation point shown in Fig. 3(a). It is clear that the peak frequency of the spectrum increased in proportion to the freestream velocity. The Strouhal number is defined by $S_{t}=f_{s} D / U_{\infty}$, where $f_{s}$ is the measured value of the peak frequency, $D$ the tube diameter, and $U_{\infty}$ the freestream velocity. The peak frequency of $62.5 \mathrm{~Hz}$ at $U_{\infty}=10.0 \mathrm{~m} / \mathrm{s}$ corresponds to the Strouhal number $S_{t}=0.2$. These characteristics agree well with the results of a bare tube ${ }^{(13)}$. It is clear that the periodic velocity fluctuation discussed above is caused by the Karman vortex shedding although the spiral fin with large pitch $1 D$ are fitted on a bare tube.

We have measured the spectra of velocity fluctuations at maximum velocity fluctuation points in the wake of other fin tubes. In Figure 3(b) the spectra in the wake of other fin tubes are expressed as $p / D=0.80,0.27,0.16$. The other high peaks are found at $63.5,59.75$, $53.25 \mathrm{~Hz}$ respectively. The dotted line in Fig. 3(b) shows the result in the wake of a bare tube. It is clear that the periodicity of Karman vortex shedding from the fin tubes become intense compared with that of a bare tube. This is caused by the asymmetry of spiral fin tubes.

In Figure 4 the peak frequencies in the wake of fin tubes are expressed as $p / D=0.80$, $0.27,0.16$. As the pitch of the fins decreases, the vortex shedding frequency decreases. It can be understood that the equivalent diameter of vortex shedding increases as the fin pitch becomes small because the fin changes the equivalent diameter of the fin tube. However, the differences between the peak frequencies of $p / D=0.80,1.00$ and that of bare tube are not clear. The large pitch of the fins causes little change of equivalent diameters of vortex shedding for these fin tubes.

From the above discussion it is concluded that the periodic velocity fluctuation caused by Karman vortex shedding is observed in the wake of the fin tube of $p / D=0.16-1.0$ although the fin is mounted spirally around a bare tube.

\subsection{Spanwise Vortex Structure}

It is known that the intensity of Aeolian tone generated from a circular cylinder depends on the spanwise correlation length of pressure fluctuation due to vortex shedding. Even in a two-dimensional flow field, Karman vortex has three-dimensional correlation structure in the spanwise direction. Therefore, it is considered that the vortex is cut easily by the fins and the vortex structure in the wake of a fin tube is different from a bare tube.

We measured the coherence function and the phase delay of velocity fluctuation. One

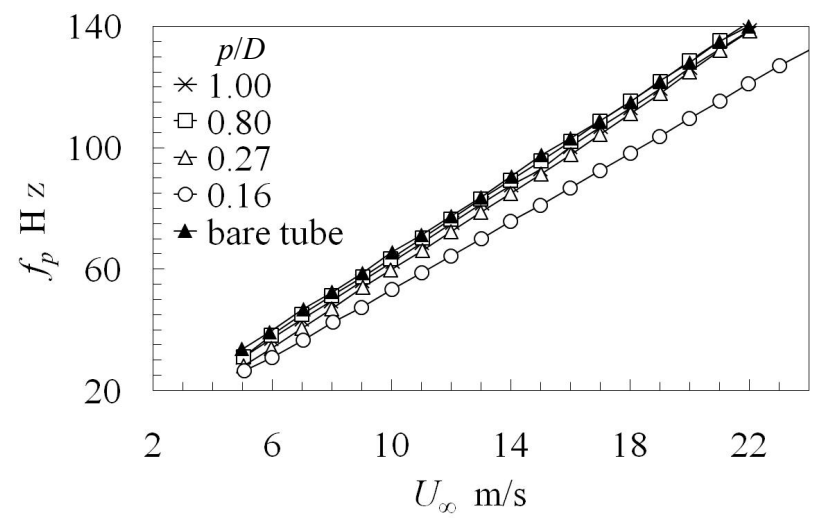

Fig. 4 Variation of peak frequencies of spectra on a function of $U_{\infty}$ 


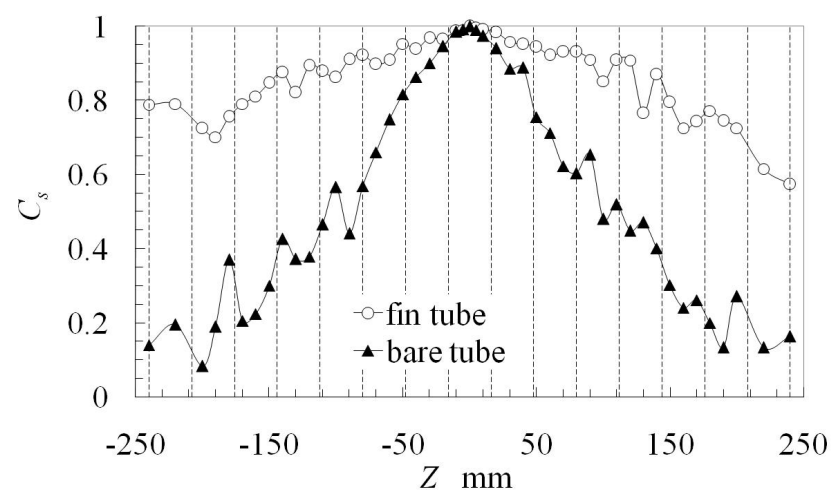

(a) Coherence of $u^{\prime}{ }_{\max }$

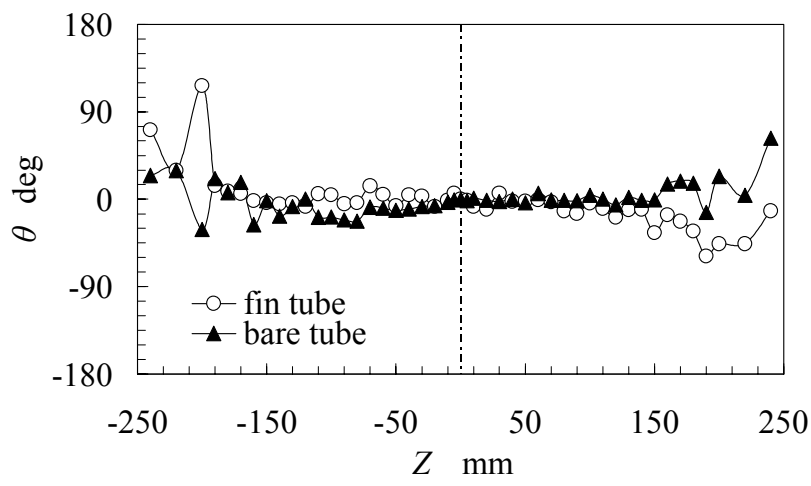

(b) Phase delay of $u_{\text {max }}^{\prime}$

Fig. 5 Spanwise distribution of coherence and phase delay $(p / D=1.00)$

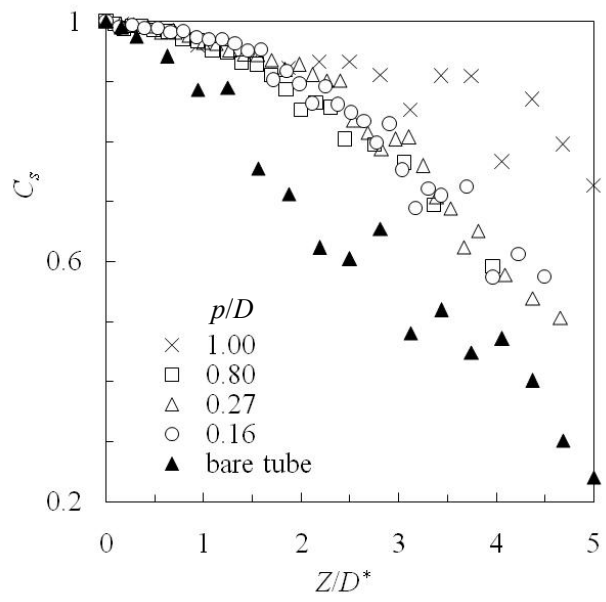

Fig. 6 Spanwise distribution of coherence of $u_{\text {max }}^{\prime}$ for $p / D=0.16-1.00$

probe was set at the reference point $(Z=0)$, where is the maximum velocity fluctuation point as shown in Figure 3(a), and the other probe was traversed along the span direction in the wake of the fin tube of $p / D=1.00$. Fig. 5(a) shows the distribution of coherence function $C_{s}$ at the vortex shedding frequency along the tube axis. The fin positions at the trailing edge of the tube are shown by the dotted lines in Fig. 5(a). The result of the fin tube is shown by open circle. It is known that the distribution of $C_{s}$ of a bare tube is fitted by the Gaussin error function as shown by closed triangle. It is understood that the fin increase the spanwise scale of vortex compared with the case of the bare tube of $D=31.8 \mathrm{~mm}$.

Figure 5(b) shows the distribution of the phase delay at the vortex shedding frequency 
measured at the maximum velocity fluctuation point along the tube axis. The result of the fin tube of $p / D=1.00$ is shown by open circle. The phase delay of the velocity fluctuation due to vortex shedding is about 0 degree from $Z=-150 \mathrm{~mm}$ to $150 \mathrm{~mm}$, which is 9.4 times of fin pitch $p$. Therefore it is considered that the vortex shedding from the fin tube has large scale structure in Z-direction regardless of the presence of the fins which has not the irregular wavy pattern like the serrated fin distribution along the tube axis ${ }^{(10)}$. The existence of a three-dimensional vortex structure corresponding to the fin position is not clear.

Figure 6 shows the effect of fin pitches on the distribution of the coherence function $C_{s}$ at the vortex shedding frequency along the tube axis. Because the coherence is distributed in symmetry in a positive and a negative direction of $Z$-axis around $Z=0$ as shown in Fig. 5(a), Fig. 6 shows only the result of a positive direction of $Z$-axis. The symbol $D^{*}$ is the equivalent diameter of vortex shedding from the fin tubes ${ }^{(13)}$. It is understood that the fins increase the spanwise vortex structure compared with the case of bare tube. The similar tendencies were obtained at other freestream velocities in the present experimental conditions.

From the above discussion it is concluded that the Karman vortex in the wake of the fin tube are not cut by the fins, and do not cause the cells structure with the scale of the pitch length of the fins.

\subsection{Flow Visualization around Fin Tube}

The flow around the fin tube of $p / D=1.00,0.80,0.27,0.16$ are visualized by using smoke wire technique to clarify the effect of the fin around a bare tube on the flow. Reynolds number, based on tube diameter $D$ and freestream velocity $U_{\infty}$, is $2.1 \times 10^{3}$.

Figures 7 (a) $\sim(\mathrm{e})$ show the flow patterns of near wake flow of $p / D=1.00$. The frame rate is 30 frames per second. It is clear that the flow patterns around the fins are similar to the case of a flat plate airfoil (Fig. 7(a), (b)). This means that the fins works as guide vanes in addition to heat transfer enhancement. Therefore, the velocities on the upper side of the fins are faster than the lower side of the fins. And the flow on the surface of the tube is inclined downward around the fin part. In the near-wake, the separating shear layers at downstream of the upper side of the fins are rolled up earlier than that of lower side (Fig. $7(\mathrm{c})$ ). As a result, the rotation axes of every vortices are inclined against the tube axis, and these vortices make the cells structure with the scale of the pitch length of the fins (Fig. 7(c), (d)). In addition, the tip leakage vortices are formed at the tips of the fins, which have the rotation axis in the flow direction, as shown in Fig. 7(d). The vortex cells seem to coalesce to the large scale vortex due to the inclination of rotation axes of the vortex cells and the tip leakage vortices (Fig. 7(e)). However these phenomena are not clear by the photograph in Fig. 7(e). The similar results are obtained at the other fin pitch of $p / D=0.80$.

Figures 8 (a) $\sim(\mathrm{f})$ show the flow patterns of $p / D=0.27$. It is found that the smoke flows between the neighboring fins along the tube axis (Fig. 8 (a), (b)). However, the flow patterns around the fins of small pitches are not clear. The cells structure of vortices with the scale of the pitch length of the fins can be observed, which is the similar result of $p / D=1.00$ (Fig. $8(\mathrm{c})$ ). The rotation axes of every vortices are inclined against the tube axis (Fig. 8 (c), (d)). In the case of a bare tube without fin, these vortex cells are not observed and the vortices are not inclined along the tube axis. Fig. 8 (e) is the photograph of other place along the tube axis at the same time of Fig. 8 (d). The spiral structure of vortex is observed at interval of smoke clearly. This means that the smoke flows spirally from upper side to lower side in the span direction. This suggests that the vortex cells are coalesced spirally to the large scale vortex (Fig. 8 (f)). Therefore it is considered that the increase of the spanwise vortex scale is caused by the increase of velocity component in the spanwise direction due to the inclination of rotation axes of the vortex cells and the tip leakage vortices. The similar results are obtained at the other fin pitch of $p / D=0.16$. 


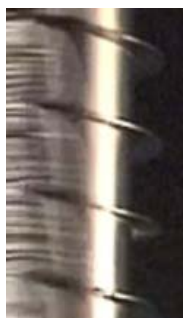

(a) $t=1 / 30 \mathrm{~s}$

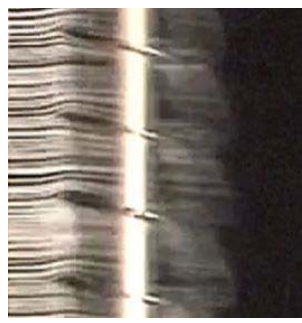

(b) $t=2 / 30 \mathrm{~s}$

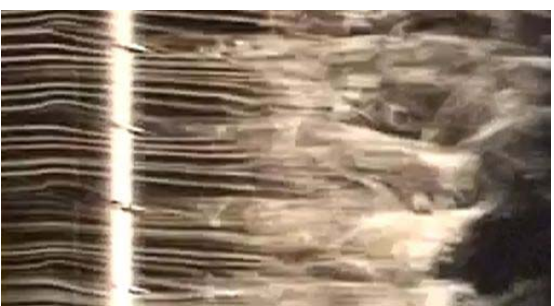

(d) $t=8 / 30 \mathrm{~s}$

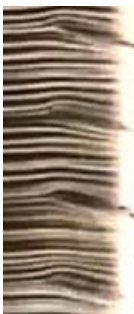

$\underline{\underline{20}}$

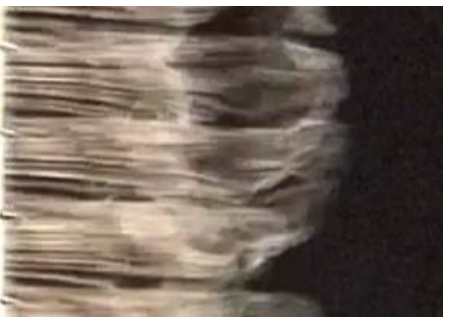

(c) $t=6 / 30 \mathrm{~s}$
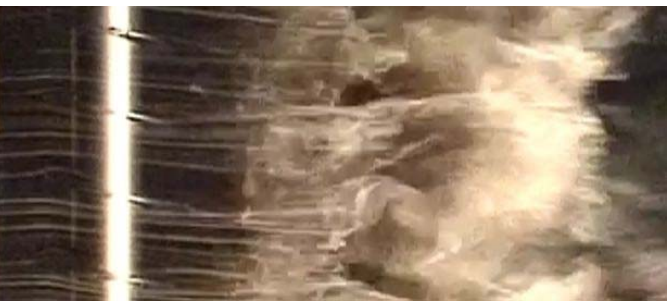

(e) $t=11 / 30 \mathrm{~s}$

Fig. 7 Flow visualization around the fin tube $(p / D=1.00)$

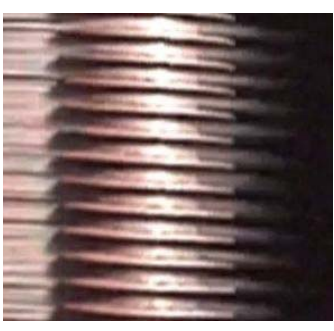

(a) $t=1 / 30 \mathrm{~s}$

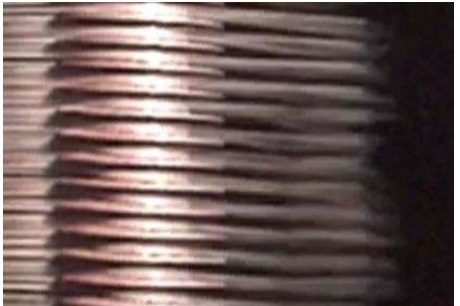

(b) $t=2 / 30 \mathrm{~s}$

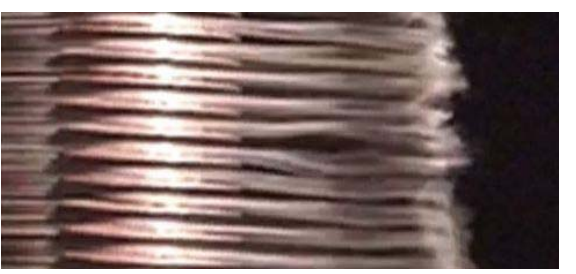

(c) $t=3 / 30 \mathrm{~s}$

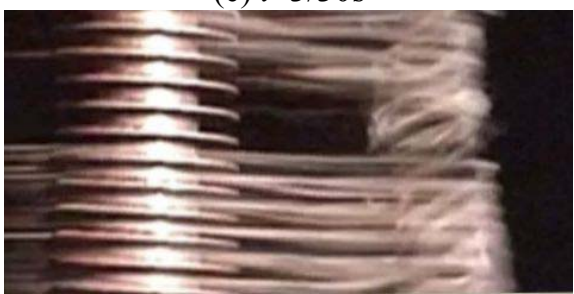

(e) $t=4 / 30 \mathrm{~s}$

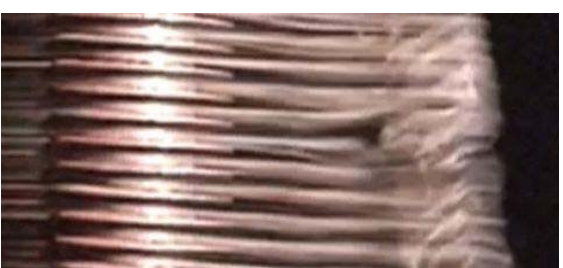

(d) $t=4 / 30 \mathrm{~s}$

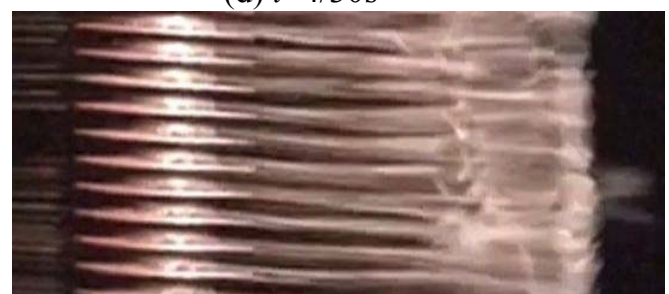

(f) $t=5 / 30 \mathrm{~s}$

Fig. 8 Flow visualization around the fin tube $(p / D=0.27)$

From the above discussion it is concluded that the vortex in the near wake of the fin tube make the cells structure, and these cells are coalesced spirally to the large scale vortex. It is considered that the maximum velocity fluctuation points in Section 3.1, 3.2 correspond to the place where these cells are coalesced spirally to the large scale vortex.

\section{Conclusions}

The flow around the fin and the vortex shedding from the fin tube were experimentally investigated. As a result following conclusions were obtained.

1. The periodic velocity fluctuation caused by Karman vortex shedding was remarkably 
observed in the wake of the fin tube although the fin was mounted spirally around a bare tube.

2. The flow on the surface of the bare tube was inclined by the fin. The spiral fin which mounted roughly on the surface of a bare tube worked as guide vanes in addition to heat transfer.

3. The vortices in the near-wake of the fin tube make the cells structure with the scale of the pitch length of the fins. The rotation axis of each vortex was inclined against the tube axis. The large scale vortex was formed by the coalescence of the vortex cells.

4. The spiral fin mounted around a bare tube has an effect of increase the spanwise correlation length of Karman vortex.

\section{Acknowledgments}

The work was partially supported by the Grant-in-Aid for Scientific Research No. 17560156 from the Japan Society for Promotion of Science (in the Japan Ministry of Education and Science) for which the authors wish to express their sincere gratitude.

\section{References}

(1)Blevins, R. D., 1986, Flow-induced Vibration, R. E. Krieger Publishing Company.

(2)Zdravkovich, M. M., 1981, Review and Classification of Various Aerodynamic and Hydrodynamic Means for Suppressing Vortex Shedding, Journal of Wind Engineering and Industrial Aerodynamics, 7, pp.145-189.

(3)Nemoto, A., Yamada, M., 1992, Flow-induced Acoustic Resonance Caused by Fin-tube Bundles, ASME PVP-Vol. 243, Symposium on Flow-induced Vibration and Noise, Vol.4, pp.137-152.

(4)Nemoto, A., Yamada, M., 1994, Flow-induced Acoustic Resonance in Staggered Tube Banks, ASME PVP-Vol. 273, Symposium on Flow-induced Vibration and Noise, pp.273-282.

(5)Nemoto, A., Takakuwa, A., Tsutsui, M., 1997, Flow- induced Acoustic Resonance with Various Finned TubeBanks, Flow-induced Vibration and Noise, ASME, Vol.2, AD-Vol.53-2, pp.311-320.

(6)Chen, Y. N., 1968, Flow-induced Vibration and Noise in Tube-bank Heat Exchangers due to Von Karman Streets, Transactions of ASME, Journal of Engineering for Industry, Series B, Vol. 94, pp. 134-146.

(7)Kouba, J., 1983, Vortex Shedding and Acoustic Emission in Finned Tube Banks Exposed to Cross Flow, ASME Pressure Vessels and Piping Conference, Vol.104, pp.213-217.

(8)Hamakawa, H., Fukano, T., Nishida, E., 2000, Vortex Shedding from Serrated-Fin-Tube Banks, 6th AIAA/CEAS Aeroacoustics Conference, Paper No.2000-2001, pp.1-8.

(9)Kawaguchi, K., Okui, K., Kawabe, Y., 2003, Vortex Generation Characteristics of Serrated Finned Tube Banks with Staggered Arrangement, Turbomachinery, Vol.3, pp.181-189.

(10)Ziada, S., Jebodhsingh, D., Weaver, D. S., Eisinger, F. L., 2005, The Effect of Fins on Vortex Shedding from a Cylinder in Cross-flow, Journal of Fluids and Structures, 21, pp.689-705.

(11)Mair, W. A., Jones, P. D. F., Palmer, R. K. W., 1975, Vortex Shedding from Finned Tubes, Journal of Sound and Vibration, Vol.39 (3), pp.293-296.

(12)Suzuki, S., Ugai, Y., Maruta, Y., Mori, S., 1986, Noise Prevention in Tube-Type Heat Exchangers Employed in Gas Turbines, Trans. Jpn. Soc. Mech. Eng., (in Japanese),

Vol.52B, No.477, pp.2137-2142.

(13)Hamakawa, H., Fukano, T., Nishida, E., Aragaki, M., 2001, Vortex Shedding from a Circular Cylinder with Fin, 7th AIAA/CEAS Aeroacoustics Conference, Paper No.2001-2215, pp.1-7. 